\title{
Integrability of the Frobenius algebra-valued Kadomtsev-Petviashvili hierarchy
}

\author{
Ian A. B. Strachan ${ }^{1, a)}$ and Dafeng Zuo $\left.2, b\right)$ \\ ${ }^{1}$ School of Mathematics and Statistics, University of Glasgow, Glasgow, United Kingdom \\ ${ }^{2}$ School of Mathematical Science, University of Science and Technology of China, \\ Hefei 230026, People's Republic of China and Wu Wen-Tsun Key Laboratory of Mathematics, \\ USTC, Chinese Academy of Sciences and School of Mathematics, China
}

(Received 14 September 2015; accepted 2 November 2015; published online 19 November 2015)

\begin{abstract}
We introduce a Frobenius algebra-valued Kadomtsev-Petviashvili (KP) hierarchy and show the existence of Frobenius algebra-valued $\tau$-function for this hierarchy. In addition, we construct its Hamiltonian structures by using the Adler-Dickey-Gelfand method. As a byproduct of these constructions, we show that the coupled KP hierarchy, defined by Casati and Ortenzi [J. Geom. Phys. 56, 418-449 (2006)], has at least $n$-"basic" different local bi-Hamiltonian structures. Finally, via the construction of the second Hamiltonian structures, we obtain some local matrix, or Frobenius algebra-valued, generalizations of classical $W$-algebras. ( 2015 AIP Publishing LLC. [http://dx.doi.org/10.1063/1.4935936]
\end{abstract}

\section{INTRODUCTION}

The Kadomtsev-Petviashvili (KP) hierarchy is defined by the set of equations

$$
\frac{\partial}{\partial t_{r}} L=\left[B_{r}, L\right], \quad r=1,2, \ldots,
$$

where $L=\partial+u_{1} \partial^{-1}+u_{2} \partial^{-2}+\cdots$ is a pseudo-differential operator with coefficients $u_{1}, u_{2}, \ldots$ being smooth functions of infinitely many variables $\mathbf{t}=\left(t_{1}, t_{2}, \ldots\right)$, with $t_{1}=x$ and $B_{r}=L_{+}^{r}$ is the pure differential part of the operator $L^{r}$ and $\partial=\frac{\partial}{\partial x}$.

A fundamental result, due to Sato, is the existence of a $\tau$-function for the KP hierarchy (see the survey ${ }^{5}$ ). Another fundamental property of this hierarchy is that it has two compatible local Hamiltonian structures. The first structure was suggested by Watanabe, ${ }^{22}$ the second by Dickey ${ }^{6}$ and, shortly after that, $\operatorname{Radul}^{18}$ proved that not only one pair of structures can be built but infinitely many. Essentially, the construction was a slight modification of the Adler-Gelfand-Dickey (AGD) method for the $n$ th-Korteweg-deVries $(\mathrm{KdV})\left(\mathrm{GD}_{n}\right)$ hierarchy in Refs. 1 and 12. We refer to Ref. 8 for a more detailed description.

Recently, there are several types of noncommutative generalizations of the KP hierarchy (see, for example, Ref. 14 and the references therein). Most of them do not preserve the above two fundamental properties. For example, the matrix $\mathrm{KP}^{3}$ has two compatible Hamiltonian structures via the AGD method, on utilizing the matrix trace map, but the second is nonlocal. Furthermore, there is no $\tau$-function for this hierarchy.

In this paper, we study certain properties of a Frobenius algebra-valued KP hierarchy. The first motivation stems from the work of Casati and Ortenzi. ${ }^{4}$ With the use of vertex operator representations of polynomial Lie algebras, they obtained a class of coupled KP hierarchy formulated as a "coupled Hirota bilinear equation." Shortly afterwards, van de Leur in Ref. 15, starting from these bilinear equations, recovered the corresponding wave functions and Lax equations with a $\mathcal{Z}_{n}$-valued Lax operator $L$, where $\mathcal{Z}_{n}=\mathbb{C}[\Lambda] /\left(\Lambda^{n}\right)$ is the maximal commutative subalgebra of $g l(n, \mathbb{R})$ and

\footnotetext{
a) E-mail address: ian.strachan@glasgow.ac.uk

b) E-mail address: dfzuo@ustc.edu.cn
} 
$\Lambda=\left(\delta_{i, j+1}\right) \in g l(m, \mathbb{R})$. A natural problem then arises as to how to construct Hamiltonian structures for these coupled KP hierarchies. The main problem in using the AGD method is that the bilinear form constructed using the usual matrix trace $\langle A, B\rangle=\operatorname{trace}(A B)$ for $A, B \in \mathcal{Z}_{n}$ is degenerate. In order to solve this, one of the current authors ${ }^{24}$ introduced a somewhat strange looking trace-type $\operatorname{map} \operatorname{tr}_{n}: g l(n, \mathbb{R}) \longrightarrow \mathbb{R}$ defined by

$$
\operatorname{tr}_{n}(A)=\operatorname{trace}\left[\left(\begin{array}{cccc}
\frac{1}{n} & \frac{1}{n-1} & \cdots & 1 \\
0 & \frac{1}{n} & \ddots & \frac{1}{2} \\
\vdots & \vdots & \ddots & \vdots \\
0 & 0 & \cdots & \frac{1}{n}
\end{array}\right) A\right]
$$

We remark that this trace-type map is not symmetric on $g l(n, \mathbb{R})$ but when restricted to the subalgebra $\mathcal{Z}_{n}$, is nondegenerate and symmetric.

Our second motivation is due to the following crucial observation. Let $\mathbf{1}_{\mathbf{n}}$ be the identity matrix and $\circ$ the matrix multiplication, then $\left\{\mathcal{Z}_{n}, \operatorname{tr}_{n}, \mathbf{1}_{\mathbf{n}}, \circ\right\}$ is a Frobenius algebra. This observation motivates us to study the $\mathcal{A}$-valued KP hierarchy via $\mathcal{A}$-valued Lax operators, where $\mathcal{A}$ is a Frobenius algebra.

This paper is organized as follows. In Section II, we will show the existence of the $\mathcal{A}$-valued $\tau$-function for the $\mathcal{A}$-KP hierarchy. In Section III, we will construct Hamiltonian structures of the $\mathcal{A}$-valued KP hierarchy. In Section IV, we will list some similar results for the $\mathcal{A}$-valued dispersionless KP hierarchy. Section V is devoted to various conclusions and a discussion of some open problems.

\section{THE FROBENIUS ALGEBRA-VALUED KP HIERARCHY AND ITS $\tau$-FUNCTION}

In this section, we will introduce an $\mathcal{A}$-valued KP hierarchy via $\mathcal{A}$-valued Lax operators and show the existence of an $\mathcal{A}$-valued $\tau$-function for the $\mathcal{A}$-KP hierarchy.

\section{A. Frobenius algebra}

We begin with the definition of a Frobenius algebra. ${ }^{9}$

Definition 2.1. A Frobenius algebra $\{\mathcal{A}, \circ, e, \omega\}$ over $\mathbb{R}$ satisfies the following conditions:

(i) $\circ: \mathcal{A} \times \mathcal{A} \rightarrow \mathcal{A}$ is a commutative, associative algebra with unity $e$;

(ii) $\omega \in \mathcal{A}^{\star}$ defines a non-degenerate inner product $\langle a, b\rangle=\omega(a \circ b)$, which is often called $a$ trace form (or Frobenius form).

Example 2.2 (Ref. 21). Let $\mathcal{A}$ be a two-dimensional commutative and associative algebra with a basis $e=e_{1}, e_{2}$ satisfying

$$
e_{1} \circ e_{1}=e_{1}, \quad e_{1} \circ e_{2}=e_{2}, \quad e_{2} \circ e_{2}=\varepsilon e_{1}+\mu e_{2}, \quad \varepsilon, \mu \in \mathbb{R},
$$

then $\mathcal{Z}_{2, k}^{\varepsilon, \mu}:=\left\{\mathcal{A}, \circ, e, \omega_{k}\right\}, k=1,2$ are Frobenius algebras, where

$$
\omega_{k}(a)=a_{k}+a_{2}\left(1-\delta_{k, 2}\right) \delta_{\varepsilon, 0}, \quad k=1,2,
$$

for $a=a_{1} e_{1}+a_{2} e_{2} \in \mathcal{A}$.

Example 2.3 (Ref. 21). Let $\mathcal{A}$ be an $n$-dimensional nonsemisimple commutative associative algebra $\mathcal{Z}_{n}$ over $\mathbb{R}$ with a unity e and a basis $e_{1}=e, \ldots, e_{n}$ satisfying

$$
e_{i} \circ e_{j}= \begin{cases}e_{i+j-1}, & i+j \leq n+1, \\ 0, & i+j=n+2 .\end{cases}
$$

Taking $\Lambda=\left(\delta_{i, j+1}\right) \in g l(m, \mathbb{R})$, one obtains a matrix representation of $\mathcal{A}$ as 


$$
e_{j} \mapsto \Lambda^{j-1}, \quad j=1, \ldots, n
$$

Similarly, for any $a=\sum_{k=1}^{n} a_{k} e_{k} \in \mathcal{A}$, we introduce $n$ trace-type forms, called "basic" trace-type forms, as follows:

$$
\omega_{k-1}(a)=a_{k}+a_{n}\left(1-\delta_{k, n}\right), \quad k=1, \ldots, n .
$$

Every trace map $\omega_{k}$ induces a nondegenerate symmetric bilinear form on $\mathcal{A}$ given by

$$
\langle a, b\rangle_{k}:=\omega_{k}(a \circ b), \quad a, b \in \mathcal{A}, \quad k=0, \ldots, n-1 .
$$

Thus, all of $\left\{\mathcal{A}, \circ, e, \omega_{k-1}\right\}$ are nonsemisimple Frobenius algebras, denoted by $\mathcal{Z}_{n, k-1}$ for $k=$ $1, \ldots, n$. We remark that the trace-type map $\operatorname{tr}_{n}$ in (1.2) is exactly a linear combination of $n$ "basic" trace-type forms as

$$
\operatorname{tr}_{n}:=\sum_{s=0}^{n-1} \omega_{s}-(n-1) \omega_{n-1}
$$

Unless otherwise stated, we assume that $\left\{\mathcal{A}, \circ, e:=\mathbf{1}_{\mathbf{n}}, \omega:=\operatorname{tr}\right\}$ is an $n$-dimensional Frobenius algebra over $\mathbb{R}$ with the basis $e_{1}=\mathbf{1}_{\mathbf{n}}, e_{2} \cdots, e_{n}$.

\section{B. The $\mathcal{A}$-valued KP hierarchy}

Let

$$
L=\mathbf{1}_{\mathbf{n}} \partial+U_{1} \partial^{-1}+U_{2} \partial^{-2}+\cdots, \quad \partial=\frac{\partial}{\partial x}
$$

be an $\mathcal{A}$-valued pseudo-differential operator ( $\Psi D O)$ with coefficients $U_{1}, U_{2}, \ldots$ being smooth $\mathcal{A}$-valued functions of an infinite many variables $t=\left(t_{1}, t_{2}, \ldots\right)$ and $t_{1}=x$.

Definition 2.4. The $\mathcal{A}-K P$ hierarchy is the set of equations

$$
\frac{\partial L}{\partial t_{r}}=\left[B_{r}, L\right]:=B_{r} \circ L-L \circ B_{r}, \quad B_{r}=L_{+}^{r}, \quad r=1,2, \ldots,
$$

where $B_{r}$ is the pure differential part of the operator $L^{r}=\underbrace{L \circ \cdots \circ L}_{r \text { terms }}$.

Generally, by imposing the constraint $\left(L^{m}\right)_{-}=0, \mathcal{A}$-KP hierarchy (2.7) reduces to the $\mathcal{A}-\mathrm{GD}_{m}$ hierarchy. The $\mathcal{A}$-KP hierarchy is equivalent to

$$
\frac{\partial B_{l}}{\partial t_{r}}-\frac{\partial B_{r}}{\partial t_{l}}+\left[B_{l}, B_{r}\right]=0
$$

Consider a case $(r=2, l=3)$, then system (2.8) becomes

$$
U_{1, t_{2}}=U_{1, x x}+2 U_{2, x}, \quad 2 U_{1, t_{3}}=2 U_{1, x x x}+3 U_{2, x x}+3 U_{2, t_{2}}+6 U_{1} \circ U_{1, x} .
$$

If we eliminate $U_{2}$ in (2.9) and rename $t_{2}=y, t_{3}=t$ and $\mathcal{U}=U_{1}$, we obtain

$$
\left(4 \mathcal{U}_{t}-12 \mathcal{U} \circ \mathcal{U}_{x}-\mathcal{U}_{x x x}\right)_{x}-3 \mathcal{U}_{y y}=0
$$

All this follows, the scalar case verbatim. But as the following example shows, when written in terms of a specific basis, this structure is broken and the underlying Frobenius algebra is hidden.

Example 2.5. Suppose that $\mathcal{A}$ is the $\mathcal{Z}_{2,2}^{\varepsilon, \mu}$ algebra and $\mathcal{U}=v e_{1}+w e_{2}$. Then, system (2.10) in component form is

$$
\left\{\begin{array}{l}
\left(4 v_{t}-12 v v_{x}-v_{x x x}-12 \varepsilon w w_{x}\right)_{x}-3 v_{y y}=0, \\
\left(4 w_{t}-12(v w)_{x}-w_{x x x}-12 \mu w w_{x}\right)_{x}-3 w_{y y}=0 .
\end{array}\right.
$$

When $\varepsilon=\mu=0$, system (2.11) reduces to the coupled KP equation (e.g., Refs. 4 and 24). Furthermore, if $v_{y}=w_{y}=0$, the coupled KP equation reduces to the coupled $K d V$ equation ${ }^{11,16,13}$ 


$$
\left\{\begin{array}{l}
4 v_{t}-12 v v_{x}-v_{x x x}=0 \\
4 w_{t}-12(v w)_{x}-w_{x x x}=0
\end{array}\right.
$$

Thus, certain multicomponent examples that have appeared in the literature are best viewed as a single $\mathcal{A}$-valued equation: writing them in terms of basis-dependent component fields obscures the underlying algebraic structure.

\section{The $\tau$-function}

Let us represent the $\mathcal{A}$-valued Lax operator $L$ in (2.6) in a dressing form

$$
L=\Phi^{-1} \circ \mathbf{1}_{\mathbf{n}} \partial \circ \Phi, \quad \Phi=\sum_{i=0}^{\infty} W_{i} \partial^{-i}, \text { with } W_{0}=\mathbf{1}_{\mathbf{n}},
$$

where the $\mathcal{A}$-valued dressing operator $\Phi$ is determined up to a multiplication on the right by $\mathbf{1}_{\mathbf{n}}+\sum_{k=1}^{\infty} C_{k} \partial^{-k}$, with arbitrary constant elements $C_{k} \in \mathcal{A}$. Then, using (2.7), we obtain

$$
\partial_{r} \Phi=-L_{-}^{r} \circ \Phi, \quad \partial_{r}=\frac{\partial}{\partial t_{r}}
$$

For simplicity, let $\xi(t, z)=\sum_{k=1}^{\infty} t_{k} z^{k}$ and $\widehat{W}(t, z)=\sum_{i=0}^{\infty} W_{i} z^{i}$, where $z \in \mathbb{C}$ is a parameter. The wave function of $\mathcal{A}$-KP hierarchy (2.7) is defined by the $\mathcal{A}$-valued function

$$
W(t, z):=\Phi e^{\xi(t, z)}=\widehat{W}(t, z) e^{\xi(t, z)} .
$$

Similarly, the adjoint wave function is given by

$$
\widetilde{W}(t, z):=\left(\Phi^{-1}\right)^{*} e^{-\xi(t, z)}=\widehat{\tilde{W}}(t, z) e^{\xi(t, z)} .
$$

Lemma 2.6. The following identities hold:

$$
\begin{aligned}
& \text { (1) } \operatorname{res}_{z}\left(\partial_{1}^{i_{1}} \cdots \partial_{k}^{i_{k}} W(t, z)\right) \circ \widetilde{W}(t, z)=0, \quad i_{j} \in \mathbb{Z}_{\geq 0}, \\
& \text { (2) } \widehat{W}(t, z)^{-1}=G(z)[\widehat{\tilde{W}}(t, z)], \\
& \text { (3) } \partial \ln \widehat{W}(t, z)=W_{1}(t)-G(z)\left[W_{1}(t)\right],
\end{aligned}
$$

where $G(z)$ is a shift operator defined by

$$
G(z)[f(t ; z, s)]=f\left(t_{1}-\frac{1}{z}, t_{2}-\frac{1}{2 z^{2}}, \ldots ; z, s\right) .
$$

Identity (2.17) is called the $\mathcal{A}$-valued bilinear identity.

Proof. (1) By definitions,

$$
L^{r} \circ W(t, z)=\left(\Phi \circ \partial \Phi^{-1}\right)^{r} \circ \Phi e^{\xi(t, z)}=z^{r} W(t, z) .
$$

Using (2.14) and (2.18), we have

$$
\partial_{r} W(t, z)=\partial_{r}\left(\Phi e^{\xi(t, z)}\right)=\left(\partial_{r} \Phi\right) e^{\xi(t, z)}+\Phi\left(\partial_{r} e^{\xi(t, z)}\right)=L_{+}^{r} W(t, z) .
$$

With (2.19), it suffices to consider only the case when all $i_{j}$ for $j>1$ vanish. Then,

$$
\operatorname{res}_{z}\left(\partial^{i} W(t, z)\right) \circ \widetilde{W}(t, z)=\operatorname{res}_{z}\left(\partial^{i} \Phi e^{x z}\right) \circ\left(\left(\Phi^{-1}\right)^{*} e^{-x z}\right)=\operatorname{res}_{\partial} \partial^{i} \Phi \circ \Phi^{-1}=0 .
$$

In the second step, we use a simple formula $\operatorname{res}_{z}\left(P e^{x z}\right) \circ\left(Q e^{-x z}\right)=\operatorname{res}_{\partial} P \circ Q^{*}$, where $P$ and $Q$ are two $\mathcal{A}$-valued $\Psi$ DOs. 
(2) Bilinear identity (2.17) implies

$$
\operatorname{res}_{z} W(t, z) \circ G(\zeta)[\widetilde{W}(t, z)]=0 .
$$

Using (2.23) and the identity $e^{\sum_{k=1}^{\infty} \frac{z^{k}}{k \zeta^{k}}}=\left(1-\frac{z}{\zeta}\right)^{-1}$, we obtain

$$
0=\operatorname{res}_{z} \widehat{W}(t, z) \circ G(\zeta)[\widehat{\tilde{W}}(t, z)]\left(1-\frac{z}{\zeta}\right)^{-1}=\zeta\left(\widehat{W}(t, \zeta) \circ G(\zeta)[\widehat{\tilde{W}}(t, z)]-\mathbf{1}_{\mathbf{n}}\right)
$$

which yields identity (2.18).

(3) Similarly, from bilinear identity (2.17), we have

$$
\operatorname{res}_{z} \partial W(t, z) \circ G(\zeta)[\widetilde{W}(t, z)]=0 .
$$

So using (2.18), we get

$$
\begin{aligned}
0 & =\operatorname{res}_{z} \partial \widehat{W}(t, z) \circ G(\zeta)[\widehat{\tilde{W}}(t, z)]\left(1-\frac{z}{\zeta}\right)^{-1} \\
& =(\partial \widehat{W}(t, \zeta)+\zeta \widehat{W}(t, \zeta)) \circ G(\zeta)[\widehat{\tilde{W}}(t, \zeta)]-\mathbf{1}_{\mathbf{n}} \zeta-W_{1}(t)+G(\zeta)\left[W_{1}(t)\right] \\
& =\partial \widehat{W}(t, \zeta) \circ \widehat{W}(t, \zeta)^{-1}-W_{1}(t)+G(\zeta)\left[W_{1}(t)\right]
\end{aligned}
$$

which implies identity (2.19).

We are now in a position to state the main theorem in this section, which can be regarded as an $\mathcal{A}$-valued counterpart of Sato's theorem ${ }^{5,7,8,19}$ for the scalar KP hierarchy.

Theorem 2.7. There is an $\mathcal{A}$-valued function $\tau=\tau(t)$ such that

$$
\widehat{W}(t, z)=G(z)[\tau(t)] \circ \tau(t)^{-1} .
$$

The $\mathcal{A}$-valued $\tau$-function is determined up to a multiplication by $C_{0} \circ e^{\sum_{k=1}^{\infty} C_{k} t_{k}}$, with arbitrary constant elements $C_{k} \in \mathcal{A}, k \in \mathbb{N}$ and arbitrary invertible constant element $C_{0} \in \mathcal{A}$.

Proof. With bilinear identity (2.17), we get

$$
\operatorname{res}_{z} W(t, z) \circ G\left(\zeta_{1}\right)\left[G\left(\zeta_{2}\right)[\widetilde{W}(t, z)]\right]=0
$$

and

$$
\operatorname{res}_{z} \widehat{W}(t, z) \circ G\left(\zeta_{1}\right)\left[G\left(\zeta_{2}\right)[\widehat{\tilde{W}}(t, z)]\right]\left(1-\frac{z}{\zeta_{1}}\right)^{-1}\left(1-\frac{z}{\zeta_{2}}\right)^{-1}=0 .
$$

It follows from (2.25) that

$$
\widehat{W}\left(t, \zeta_{1}\right) \circ G\left(\zeta_{2}\right)\left[G\left(\zeta_{1}\right)\left[\widehat{\tilde{W}}\left(t, \zeta_{1}\right)\right]\right]=\widehat{W}\left(t, \zeta_{2}\right) \circ G\left(\zeta_{1}\right)\left[G\left(\zeta_{2}\right)\left[\widehat{\tilde{W}}\left(t, \zeta_{2}\right)\right]\right],
$$

which becomes, using (2.18),

$$
\widehat{W}\left(t, \zeta_{1}\right) \circ G\left(\zeta_{2}\right)\left[\widehat{W}\left(t, \zeta_{1}\right)^{-1}\right]=\widehat{W}\left(t, \zeta_{2}\right) \circ G\left(\zeta_{1}\right)\left[\widehat{W}\left(t, \zeta_{2}\right)^{-1}\right] .
$$

Letting $\mu(t, z)=\ln \widehat{W}(t, z)$ and taking into account (2.26), we have

$$
\mu\left(t, \zeta_{1}\right)-G\left(\zeta_{2}\right)\left[\mu\left(t, \zeta_{1}\right)\right]=\mu\left(t, \zeta_{2}\right)-G\left(\zeta_{1}\right)\left[\mu\left(t, \zeta_{2}\right)\right] .
$$

For simplicity, we denote

$$
N(z):=\frac{\partial}{\partial z}-\sum_{k=1}^{\infty} z^{-k-1} \partial_{k}, \quad B_{i}:=\operatorname{res}_{z} z^{i} N(z) \mu(t, z) .
$$

Applying the operator to (2.27) after renaming $\zeta_{1}=z$ and $\zeta_{2}=\zeta$, we get

$$
N(z) \mu(t, z)-G(\zeta)[N(z) \mu(t, z)]=-\sum_{k=1}^{\infty} z^{-k-1} \partial_{k} \mu(t, \zeta) .
$$

Multiplying by $z^{i}$ on both sides of (2.28) and taking the residues res $_{z}$, we obtain

$$
B_{i}=G(\zeta)\left[B_{i}\right]-\partial_{i} \mu(t, z)
$$


and furthermore,

$$
\partial_{j} B_{i}-\partial_{i} B_{j}=G(\zeta)\left[\partial_{j} B_{i}-\partial_{i} B_{j}\right]
$$

which yields $\partial_{j} B_{i}-\partial_{i} B_{j}=$ const $\in \mathcal{A}$. The left side of (2.30) is a differential polynomial in $W_{i}(t)$ without constant terms, we thus have $\partial_{j} B_{i}=\partial_{i} B_{j}$. So, there is an $\mathcal{A}$-valued function $\tau=\tau(t)$ such that $B_{i}=\partial_{i} \ln \tau$. By using (2.29), we get

$$
\partial_{i} \mu(t, z)=\partial_{i}(G(\zeta)[\ln \tau]-\ln \tau)
$$

which yields (2.24). The rest of the theorem is obvious.

Corollary 2.8. For any $i \in \mathbb{N}$, the following identity holds:

$$
\operatorname{res} L^{i}=\frac{\partial}{\partial t_{i}}\left(\tau_{x} \circ \tau^{-1}\right) \text {. }
$$

Proof. Equating the residue on both sides of (2.14), we have

$$
\operatorname{res} L^{i}=-\partial_{i} W_{1}(t) \text {. }
$$

Observe that $\operatorname{res}_{z} z^{i} N(z) \mu(t, z)=B_{i}=\partial_{i} \ln \tau$ and $\mu(t, z)=\ln \widehat{W}(t, z)$, then we get

$$
\begin{aligned}
\frac{\partial}{\partial t_{i}}\left(\tau_{x} \circ \tau^{-1}\right) & =\partial \partial_{i} \ln \tau=\operatorname{res}_{z} z^{i} N(z) \partial \ln \widehat{W}(t, z) \quad \text { using (2.19) } \\
& =\operatorname{res}_{z} z^{i} N(z)\left(W_{1}(t)-G(z)\left[W_{1}(t)\right]\right)=\operatorname{res}_{z} z^{i} N(z) W_{1}(t) \\
& =\operatorname{res}_{z} z^{i} \sum_{k=1}^{\infty} z^{-k-1} \partial_{k} W_{1}(t)=-\partial_{i} W_{1}(t) .
\end{aligned}
$$

Taking into account (2.32), we obtain desired formula (2.31).

Example 2.9. Let $A \in \mathcal{A}$ be a constant element, then

$$
\tau=\mathbf{1}_{n}+\exp \left(2 A x+2 A^{3} t\right)
$$

is an $\mathcal{A}$-valued $\tau$-function of the $\mathcal{A}$-valued KdV equation $4 \mathcal{U}_{t}-12 \mathcal{U} \circ \mathcal{U}_{x}-\mathcal{U}_{x x x}=0$.

Taking $\mathcal{A}$ to be the Frobenius algebra $\mathcal{Z}_{2}$, the $\mathcal{A}$-valued KdV equation is exactly coupled KdV equations (2.12). By choosing $A=\left(\begin{array}{ll}a & 0 \\ b & a\end{array}\right) \in \mathcal{A}$, we then have

$$
\tau=\left(\begin{array}{cc}
1+\exp \left(2 a x+2 a^{3} t\right) & 0 \\
\left(2 b x+2 b^{3} t\right) \exp \left(2 a x+2 a^{3} t\right) & 1+\exp \left(2 a x+2 a^{3} t\right)
\end{array}\right):=\left(\begin{array}{cc}
\tau_{0} & 0 \\
\tau_{1} & \tau_{0}
\end{array}\right)
$$

and

$$
\left(\begin{array}{ll}
v & 0 \\
w & v
\end{array}\right)=\mathcal{U}=\frac{\partial}{\partial x}\left(\tau_{x} \tau^{-1}\right)=\left(\begin{array}{cc}
\left(\log \tau_{0}\right)_{x x} & 0 \\
\left(\frac{\tau_{1}}{\tau_{0}}\right)_{x x} & \left(\log \tau_{0}\right)_{x x}
\end{array}\right) .
$$

Thus, we obtain a solution of couple KdV equation (2.12) given by

$$
v=\left(\log \tau_{0}\right)_{x x}, \quad w=\left(\frac{\tau_{1}}{\tau_{0}}\right)_{x x} .
$$

We remark that variable transformation (2.33) has been used to derive the coupled KdV equation from the Hirota equation in Ref. 4. The form of this (i.e., Equation (2.33)) may thus be traced back to the nilpotent elements that appear in the Frobenius algebra $\mathcal{A}$.

\section{HAMILTONIAN STRUCTURES OF THE $\mathcal{A}$-VALUED KP HIERARCHY}

In this section, we will use the AGD-scheme (e.g., Refs. 1, 12, and 8) to construct Hamiltonian structures of the $\mathcal{A}$-KP hierarchy. For the clarity, let $P=\sum_{i} P_{i} \partial^{i}$ be an $\mathcal{A}$-valued $\Psi D O$, in what follows, we denote $P_{+}$the pure differential part of the operator $P$ and 


$$
P_{-}=P-P_{+}, \quad \operatorname{res}(P)=P_{-1}, \quad P^{*}=\sum_{i}(-1)^{i} \partial^{i} P_{i}
$$

Lemma 3.1. Suppose A and B are two $\mathcal{A}$-valued $\Psi$ DOs, then

$$
\operatorname{tr} \int \operatorname{res} A \circ B d x=\operatorname{tr} \int \operatorname{res} B \circ A d x \text {. }
$$

Proof. We first show that

$$
\operatorname{res}[A, B]=\frac{\partial h(x, \mathbf{t})}{\partial x},
$$

where $h(x, \mathbf{t})$ is a certain $\mathcal{A}$-valued function. By linearity, it is sufficient to prove (3.1) for any two $\mathcal{A}$-valued monomials $A=A_{i} \partial^{i}, B=B_{j} \partial^{j}$. If $i, j \geq 0$ or $i+j<1$, then res $[A, B]=0$ and so, $h=0$. We thus only need consider the case $i \geq 0, j<0$ and $i+j \geq 1$. A direct computation gives

$$
\begin{aligned}
\operatorname{res}[A, B] & =C_{i}^{i+j+1}\left(A_{i} \circ B_{j}^{(i+j+1)}+(-1)^{i+j} B_{j} \circ A_{i}^{(i+j+1)}\right) \\
& =\frac{\partial}{\partial x}\left(C_{i}^{i+j+1} \sum_{s=0}^{i+j}(-1)^{s} A_{i}^{(s)} \circ B_{j}^{i+j-s}\right):=\frac{\partial}{\partial x} h .
\end{aligned}
$$

Obviously, $h$ is $\mathcal{A}$-valued. Furthermore, taking the trace form tr on both sides of (3.2), we obtain $\operatorname{tr} \operatorname{res}[A, B]=\operatorname{tr} \frac{\partial h}{\partial x}$. With this, identity (3.1) follows immediately.

\section{A. Case $U_{0} \neq 0$, i.e., $V_{m-1} \neq 0$}

Let $L=\mathbf{1}_{\mathbf{n}} \partial+U_{0}+U_{1} \partial^{-1}+U_{2} \partial^{-2}+\cdots$ be an $\mathcal{A}$-valued $\Psi D O$ with an additional term $U_{0}$. Denoting

$$
\mathcal{L}:=L^{m}=\mathbf{1}_{\mathbf{n}} \partial^{m}+V_{m-1} \partial^{m-1}+V_{m-2} \partial^{m-2}+\cdots, \quad V_{i}=\sum_{q=1}^{n} v_{[i] q} e_{q} .
$$

In the following, our Hamiltonian structures will be established in terms of the "dynamical coordinates" $\left\{v_{[i] q}\right\}$.

We denote by $\mathfrak{D}$ the differential algebra of polynomials in formal symbols $\left\{v_{[i] q}^{(j)}\right\}$, where $v_{[i] q}^{(j)}=\frac{\partial^{j} v_{[i] q}}{\partial x^{j}}$ for $q=1, \ldots, n$ and $j=0,1, \ldots$ We consider a subalgebra $\mathcal{D}$ of $\mathfrak{D}$ with the element of the form $\operatorname{tr} F(V)$, where $F(V)$ is an $\mathcal{A}$-valued differential polynomial with respect to its arguments $V_{i}$. We denote the space of functionals by

$$
\widetilde{\mathcal{D}}=\left\{\tilde{f}=\int \operatorname{tr} F(V) d x \mid \operatorname{tr} F(V) \in \mathcal{D}\right\} .
$$

The variational derivative with respect to an algebra-valued field has been discussed in Ref. 17 . In the present context, for $V=\sum_{q=1}^{n} v_{q} e_{q}$, the variational derivative $\frac{\delta F}{\delta V}$ is defined by

$$
\tilde{f}(v+\delta v)-\tilde{f}(v)=\int \operatorname{tr}\left(\frac{\delta F}{\delta V} \circ \delta V+o(\delta V)\right) d x=\int \sum_{q=1}^{n}\left(\frac{\delta f}{\delta v_{q}} \delta v_{q}+o(\delta v)\right) d x
$$

where $f(v)=\operatorname{tr} F(V), \delta V=\sum_{q=1}^{n} \delta v_{q} e_{q} \in \mathcal{A}$, and $\frac{\delta f}{\delta v_{q}}=\sum_{j=0}^{\infty}(-\partial)^{j} \frac{\partial f}{\partial v_{q}^{(j)}}$. Without confusion, we use the notation $\frac{\delta f}{\delta V}$ instead of $\frac{\delta F}{\delta V}$.

Suppose $\mathbf{a}=\left(a_{m-1}, a_{m-2}, \ldots\right)$ with elements

$$
a_{i}=\sum_{q=1}^{n} a_{[i] q} e_{q} \in \mathcal{A}, \quad i=m-1, m-2, \ldots .
$$


We define a vector field associated to $\mathbf{a}$ by the formula

$$
\partial_{\mathbf{a}}=\sum_{i=-\infty}^{m-1} \sum_{j=0}^{\infty} \sum_{q=1}^{n} a_{[i] q}^{(j)} \frac{\partial}{\partial v_{[i] q}^{(j)}} .
$$

Obviously, $\partial_{\mathbf{a}}$ and $\partial$ commute, i.e.,

$$
\partial \partial_{\mathbf{a}} f=\partial_{\mathbf{a}} \partial f, \quad \text { for } f \in \mathcal{D} .
$$

The set of all vector fields $\partial_{\mathbf{a}}$ will be denoted by $\mathcal{V}$, which is a Lie algebra with respect to the commutator $\left[\partial_{\mathbf{a}}, \partial_{\mathbf{b}}\right]=\partial_{\partial_{\mathbf{a}} \mathbf{b}-\partial_{\mathbf{b}} \mathbf{a}}$. Let $\Omega^{1}$ be the dual space of $\mathcal{V}$ consisting of formal $\mathcal{A}$-valued integral operators

$$
X=\sum_{i=-\infty}^{m-1} \partial^{-i-1} X_{i}, \quad X_{i} \in \mathcal{A}
$$

with the pairing

$$
\left\langle\partial_{\mathbf{a}}, X\right\rangle=\langle\mathbf{a}, X\rangle=\operatorname{tr} \int \operatorname{res}(\mathbf{a} \circ X) d x .
$$

With the use of formulae (3.4) and (3.6), the action of $\mathcal{V}$ on $\mathcal{D}$ can be transferred to $\widetilde{\mathcal{D}}$,

$$
\partial_{\mathbf{a}} \tilde{f}=\partial_{\mathbf{a}} \int f d x=\int \partial_{\mathbf{a}} f d x=\sum_{i=-\infty}^{m-1} \sum_{q=1}^{n} \int \frac{\delta f}{\delta v_{[i] q}} a_{[i] q} d x=\operatorname{tr} \int \sum_{i=-\infty}^{m-1} a_{i} \circ \frac{\delta f}{\delta V_{i}} d x .
$$

If we set

$$
\frac{\delta f}{\delta \mathcal{L}}=\sum_{i=-\infty}^{m-1} \partial^{-i-1} \frac{\delta f}{\delta V_{i}}
$$

and identify the vector $\mathbf{a}=\left(a_{n-1}, a_{n-2}, \ldots\right)$ with the $\mathcal{A}$-valued $\Psi$ DO $\mathbf{a}=\sum_{i=-\infty}^{m-1} a_{i} \partial^{i}$, we then have

$$
\partial_{\mathbf{a}} \tilde{f}=\operatorname{tr} \int \operatorname{res}\left(\mathbf{a} \circ \frac{\delta f}{\delta \mathcal{L}}\right) d x,
$$

which follows

$$
\left\langle\partial_{\mathbf{a}}, \frac{\delta f}{\delta \mathcal{L}}\right\rangle=\partial_{\mathbf{a}} \tilde{f}=\left\langle\partial_{\mathbf{a}}, d \tilde{f}\right\rangle, \quad d \tilde{f}=\frac{\delta f}{\delta \mathcal{L}} \in \Omega^{1}
$$

Lemma 3.2. The mapping $\mathcal{H}: \Omega^{1} \rightarrow \mathcal{V}$ defined by $\mathcal{H}(X)=\partial_{A^{(z)}(X)}$ is a Hamiltonian mapping, ${ }^{28}$ where

$$
A^{(z)}(X)=(\widetilde{\mathcal{L}} \circ X)_{+} \circ \tilde{\mathcal{L}}-\tilde{\mathcal{L}} \circ(X \circ \widetilde{\mathcal{L}})_{+}
$$

and $\widetilde{\mathcal{L}}=\mathcal{L}-z$ and $z$ is an arbitrary parameter.

Proof. When the Frobenius algebra $\mathcal{A}$ is taken to be $\mathbb{R}$, this mapping is the famous Adler mapping which is a Hamiltonian mapping. For a general commutative Frobenius algebra, its trace form is nondegenerate and symmetric. We thus follow the same ideas as used in Ref. 6 to obtain the proof by replacing the scalar operators by $\mathcal{A}$-valued operators.

We rewrite $A^{(z)}(X)$ in (3.11) as

$$
A^{(z)}(X)=H^{m(0)}(X)+z H^{m(\infty)}(X)
$$

that is to say,

$$
H^{m(0)}(X)=(\mathcal{L} \circ X)_{+} \circ \mathcal{L}-\mathcal{L} \circ(X \circ \mathcal{L})_{+}, \quad H^{m(\infty)}(X)=\left[\mathcal{L}_{-}, X_{+}\right]_{-}-\left[\mathcal{L}_{+}, X_{-}\right]_{+} .
$$

By using Lemma 3.2, $H^{m(0)}$ and $H^{m(\infty)}$ are Hamiltonian mappings. We thus get two compatible Poisson brackets of the $\mathcal{A}-\mathrm{KP}$ hierarchy associated with $\mathcal{L}:=L^{m}$ are given by 


$$
\begin{aligned}
\{\tilde{f}, \tilde{g}\}^{m(\infty)} & =\operatorname{tr} \int \operatorname{res} H^{m(\infty)}\left(\frac{\delta f}{\delta \mathcal{L}}\right) \circ \frac{\delta g}{\delta \mathcal{L}} d x \\
& =\operatorname{tr} \int \operatorname{res}\left(\left[\mathcal{L}_{-},\left(\frac{\delta f}{\delta \mathcal{L}}\right)_{+}\right]_{-}-\left[\mathcal{L}_{+},\left(\frac{\delta f}{\delta \mathcal{L}}\right)\right]_{-}\right]_{+} \circ \frac{\delta g}{\delta \mathcal{L}} d x
\end{aligned}
$$

and

$$
\begin{aligned}
\{\tilde{f}, \tilde{g}\}^{m(0)} & =\operatorname{tr} \int \operatorname{res} H^{m(0)}\left(\frac{\delta f}{\delta \mathcal{L}}\right) \circ \frac{\delta g}{\delta \mathcal{L}} d x \\
& =\operatorname{tr} \int \operatorname{res}\left(\left(\mathcal{L} \circ \frac{\delta f}{\delta \mathcal{L}}\right)_{+} \circ \mathcal{L}-\mathcal{L} \circ\left(\frac{\delta f}{\delta \mathcal{L}} \circ \mathcal{L}\right)_{+}\right) \circ \frac{\delta g}{\delta \mathcal{L}} d x,
\end{aligned}
$$

where $\tilde{f}, \tilde{g}$ are two functionals. Furthermore, we have the following.

Theorem 3.3. The $\mathcal{A}-K P$ hierarchy $\frac{\partial L}{\partial t_{r}}=\left[B_{r}, L\right]$ admits a bi-Hamiltonian representation given by

$$
\frac{\partial \mathcal{L}}{\partial t_{r}}=H^{m(0)}\left(\frac{\delta h_{r}}{\delta \mathcal{L}}\right)=H^{m(\infty)}\left(\frac{\delta g_{r}}{\delta \mathcal{L}}\right),
$$

with the Hamiltonians

$$
\tilde{h}_{r}=\frac{m}{r} \operatorname{tr} \int \operatorname{res} L^{r} d x \quad \text { and } \quad \tilde{g}_{r}=-\frac{m}{r+m} \operatorname{tr} \int \operatorname{res} L^{m+r} d x .
$$

Proof. Observe that the $\mathcal{A}-\mathrm{KP}$ hierarchy $\frac{\partial L}{\partial t_{r}}=\left[B_{r}, L\right]$ is equivalent to $\frac{\partial \mathcal{L}}{\partial t_{r}}=\left[B_{r}, \mathcal{L}\right]$. By definition in (3.4), one obtains

$$
\frac{\delta}{\delta \mathcal{L}} \operatorname{tr} \int \operatorname{res} L^{r} d x=\frac{r}{m} L^{r-m}
$$

With the help of (3.12), one thus gets

$$
H^{m(0)}\left(\frac{\delta h_{r}}{\delta \mathcal{L}}\right)=H^{m(0)}\left(L^{r-m}\right)=\left[B_{r}, \mathcal{L}\right]
$$

and

$$
\begin{aligned}
H^{m(\infty)}\left(\frac{\delta g_{r}}{\delta \mathcal{L}}\right) & =-H^{m(\infty)}\left(L^{r}\right)=\left[\mathcal{L}_{+}, L_{-}^{r}\right]_{+}-\left[\mathcal{L}_{-}, L_{+}^{r}\right]_{-} \\
& =\left[\mathcal{L}, L_{-}^{r}\right]_{+}-\left[\mathcal{L}, L_{+}^{r}\right]_{-}=\left[B_{r}, \mathcal{L}\right],
\end{aligned}
$$

which yields this theorem.

\section{B. Case $U_{0}=0$, i.e., $V_{m-1}=0$}

If we restrict to $V_{m-1}=0$, it is easy to check that the first Hamiltonian structure automatically reduces to this submanifold, but the second one is reducible if and only if

$$
\operatorname{res}\left[\mathcal{L}, \frac{\delta f}{\delta \mathcal{L}}\right]=0
$$

which is equivalent to the condition

$$
X_{m-1}=\frac{1}{m} \sum_{i=-\infty}^{m-2}\left(\left(\begin{array}{r}
-i-1 \\
m-i
\end{array}\right) X_{i}^{(m-i-1)}+\sum_{j=i+1}^{m-1}\left(\begin{array}{r}
-i-1 \\
j-i
\end{array}\right)\left(X_{i} \circ V_{j}\right)^{(j-i-1)}\right),
$$

where $X_{i}=\frac{\delta f}{\delta V_{i}} \in \mathcal{A}$. We denote the corresponding reduced brackets by $\{,\}^{m(\infty)}$ and $\{,\}_{D}^{m(0)}$.

Corollary 3.4. The coupled KP hierarchy defined in Ref. 4 has at least n "basic" different local bi-Hamiltonian structures.

Proof. As explained in the Introduction, the coupled KP hierarchy defined in Refs. 4 and 15 is exactly the $\mathcal{Z}_{n}$-KP hierarchy. According to Example 2.3, the algebra $\mathcal{Z}_{n}$ has at least $n$-"basic" 
different ways to be realized as the Frobenius algebra. With this, the corollary follows immediately from Theorem 3.3.

Definition 3.5. In terms of the basis $\left\{v_{[i] q}\right\}$, the second Poisson bracket $\{,\}^{m(0)}$ for $L^{m}$ in (3.3) and the reduced bracket $\{,\}_{D}^{m(0)}$ for $L^{m}$ with the constraint $V_{m-1}=0$ will provide two kinds of local W-type algebras, we call them the $W_{\mathcal{A} \mathbb{K} \mathbb{P}}^{(n, m)}$-algebra and the $W_{\infty}^{(n, m)}$-algebra, respectively. Under the reduction $L_{-}^{m}=0$, the corresponding algebras are called the $W_{\mathcal{A G D}}^{(n, m)}$-algebra and the $W_{(n, m)}$-algebra, respectively.

With the use of (3.3) and (3.17), one knows that all of them are local matrix generalizations of $W$-algebras. To conclude this section, two examples will be given to illustrate our construction.

Example 3.6. Consider the $\mathcal{A}-K d V$ hierarchy with the Lax operator $L^{2}=\mathbf{1}_{\mathbf{n}} \partial^{2}+V$, i.e., $\quad L_{-}^{2}=$ 0 . We denote $X=\partial^{-2} X_{1}+\partial^{-1} X_{0}$ and $Y=\partial^{-2} Y_{1}+\partial^{-1} Y_{0}$. Condition (3.16) becomes $X_{1}=\frac{1}{2} X_{0}^{\prime}$, then we have

$$
H^{2(\infty)}=\left[X, L^{2}\right]_{+}=-2 X_{0}^{\prime}
$$

and

$$
H^{2(0)}(X)=\left(L^{2} \circ X\right)_{+} \circ L^{2}-L^{2} \circ\left(X \circ L^{2}\right)_{+}=2 V \circ X_{0}^{\prime}+X_{0} \circ V^{\prime}+\frac{1}{2} X_{0}^{\prime \prime \prime} .
$$

Thus, two compatible Poisson brackets of the $\mathcal{A}-K d V$ hierarchy ${ }^{25}$ are given by

and

$$
\{\tilde{f}, \tilde{g}\}^{2(\infty)}=2 \operatorname{tr} \int \frac{\delta f}{\delta V} \circ \frac{\partial}{\partial x} \frac{\delta g}{\delta V} d x
$$

$$
\{\tilde{f}, \tilde{g}\}_{D}^{2(0)}=-\frac{1}{2} \operatorname{tr} \int \frac{\delta f}{\delta V} \circ\left(\mathbf{1}_{\mathbf{n}} \frac{\partial^{3}}{\partial x^{3}}+2 V \frac{\partial}{\partial x}+2 \frac{\partial}{\partial x} V\right) \circ \frac{\delta g}{\delta V} d x .
$$

In particular, if one chooses the algebra $\mathcal{A}$ to be the algebra $\mathcal{Z}_{2}$ defined in Example 2.3, one obtains the $\mathcal{Z}_{2}-K d V$ equation for $V=v e_{1}+$ we 2 given by

$$
4 v_{t}-12 v v_{x}-v_{x x x}=0, \quad 4 w_{t}-12(v w)_{x}-w_{x x x}=0 .
$$

According to Corollary 3.4, system (3.18) can be written as

$$
\left(\begin{array}{l}
v \\
w
\end{array}\right)_{t}=\left(\begin{array}{ll}
0 & \partial \\
\partial & 0
\end{array}\right)\left(\begin{array}{l}
\frac{\delta H_{2}}{\delta v} \\
\frac{\delta H_{2}}{\delta w}
\end{array}\right)=\left(\begin{array}{ll}
0 & J_{0} \\
J_{0} & J_{1}
\end{array}\right)\left(\begin{array}{l}
\frac{\delta H_{1}}{\delta v} \\
\frac{\delta H_{1}}{\delta w}
\end{array}\right)
$$

with Hamiltonians

$$
H_{1}=\int_{\mathbb{S} 1} v w d x, \quad H_{2}=\int_{\mathbb{S} 1}\left(\frac{3}{2} v^{2} w+\frac{1}{4} v w_{x x}\right) d x
$$

and

with Hamiltonians

$$
\left(\begin{array}{c}
v \\
w
\end{array}\right)_{t}=\left(\begin{array}{cc}
0 & \partial \\
\partial & -\partial
\end{array}\right)\left(\begin{array}{l}
\frac{\delta \widetilde{H}_{2}}{\delta v} \\
\frac{\delta \widetilde{H}_{2}}{\delta w}
\end{array}\right)=\left(\begin{array}{cc}
0 & J_{0} \\
J_{0} & J_{1}-J_{0}
\end{array}\right)\left(\begin{array}{c}
\frac{\delta \widetilde{H}_{1}}{\delta v} \\
\frac{\delta \widetilde{H}_{1}}{\delta w}
\end{array}\right)
$$

$$
\widetilde{H}_{1}=\int_{\mathbb{S} 1}\left(\frac{1}{2} v^{2}+v w\right) d x, \quad \widetilde{H}_{2}=\int_{\mathbb{S} 1}\left(\frac{3}{2} v^{2} w+\frac{1}{4} v w_{x x}+\frac{1}{2} v^{3}+\frac{1}{8} v v_{x x}\right) d x,
$$

where $J_{0}=\frac{1}{4} \partial^{3}+v \partial+\partial v$ and $J_{1}=w \partial+\partial w$.

Example 3.7 (The $\mathcal{A}$-Boussinesq hierarchy). In this case, we have $\mathcal{L}=\mathrm{I}_{m} \partial^{3}+V_{1} \partial+V_{0}$. Let us take $\tilde{f}, \tilde{g} \in \widetilde{\mathcal{D}}$ and denote

$$
X_{j}=\frac{\delta f}{\delta V_{j}}, \quad Y_{j}=\frac{\delta g}{\delta V_{j}}, \quad j=0,1 .
$$


Using condition (3.17), we have

$$
\frac{\delta f}{\delta \mathcal{L}}=\partial^{-3} X_{2}+\partial^{-2} X_{1}+\partial^{-1} X_{0}, \quad \frac{\delta g}{\delta \mathcal{L}}=\partial^{-3} Y_{2}+\partial^{-2} Y_{1}+\partial^{-1} Y_{0},
$$

where $X_{2}=X_{1}^{\prime}-\frac{1}{3} X_{0}^{\prime \prime}-\frac{1}{3} X_{0} V_{1}$ and $Y_{2}=Y_{1}^{\prime}-\frac{1}{3} Y_{0}^{\prime \prime}-\frac{1}{3} Y_{0} V_{1}$.

A direct calculation gives two Poisson brackets of the $\mathcal{A}$-Boussinesq hierarchy

$$
\{\tilde{f}, \tilde{g}\}^{3(\infty)}=3 \operatorname{tr} \int\left(X_{1} Y_{0}^{\prime}+X_{0} Y_{1}^{\prime}\right) d x
$$

and

$$
\begin{aligned}
\{\tilde{f}, \tilde{g}\}_{D}^{3(0)} & =\operatorname{tr} \int\left(\frac{2}{3} X_{0} Y_{0}^{(5)}-X_{0} Y_{1}^{(4)}+X_{1} Y_{0}^{(4)}-2 X_{1} Y_{1}^{(3)}\right) d x \\
& +\operatorname{tr} \int\left(\frac{1}{3} X_{0} Y_{0}^{\prime}-\frac{1}{3} X_{0}^{\prime} Y_{0}\right) V_{1}^{2} d x \\
& +\operatorname{tr} \int\left(\frac{2}{3} X_{0} Y_{0}^{(3)}-\frac{2}{3} X_{0}^{(3)} Y_{0}+X_{1}^{\prime \prime} Y_{0}-X_{0} Y_{1}^{\prime \prime}+X_{1}^{\prime} Y_{1}-X_{1} Y_{1}^{\prime}\right) V_{1} d x \\
& +\operatorname{tr} \int\left(X_{0} Y_{0}^{\prime \prime}-X_{0}^{\prime \prime} Y_{0}+2 X_{1}^{\prime} Y_{0}-X_{1} Y_{0}^{\prime}+X_{0}^{\prime} Y_{1}-2 X_{0} Y_{1}^{\prime}\right) V_{0} d x
\end{aligned}
$$

More specifically, by analogy to the classical W-algebra in Refs. 2 and 10, we set

$$
W_{2}=V_{1}, \quad W_{3}=V_{0}-\frac{1}{2} V_{1}^{\prime},
$$

then for any two $\mathcal{A}$-valued test functions $F$ and $G$, we have

$$
\begin{gathered}
\left\{\operatorname{tr} \int F W_{2} d x, \operatorname{tr} \int G W_{2} d x\right\}_{D}^{3(0)}=\operatorname{tr} \int\left(2 F^{(3)}+2 W_{2} F^{\prime}+W_{2}^{\prime} F\right) G d x, \\
\left\{\operatorname{tr} \int F W_{2} d x, \operatorname{tr} \int G W_{3} d x\right\}_{D}^{3(0)}=\operatorname{tr} \int\left(3 W_{3} F^{\prime}+W_{3}^{\prime} F\right) G d x,
\end{gathered}
$$

and

$$
\begin{aligned}
& \left\{\operatorname{tr} \int F W_{3} d x, \operatorname{tr} \int G W_{3} d x\right\}_{D}^{3(0)}=\frac{1}{6} \operatorname{tr} \int\left(\left(2 F G^{\prime}-2 F^{\prime} G\right) W_{2}^{2}\right. \\
+ & \left.F G^{(5)}\right) d x+\frac{1}{12} \operatorname{tr} \int\left(2 F G^{(3)}-2 F^{(3)} G+3 F^{\prime \prime} G^{\prime}-3 F^{\prime} G^{\prime \prime}\right) W_{2} d x .
\end{aligned}
$$

We thus confirm that $W_{\mathbf{k}}$ for $\mathbf{k}=2,3$ are spin-k conformally primary $\mathcal{A}$-valued fields. But notice that the equation $\operatorname{tr} F W_{2}^{2}=\left(\operatorname{tr} F W_{2}\right)^{2}$ has no $\mathcal{A}$-valued non-zero solution, which means the classical $W_{3}$-algebra is not a subalgebra of the $W_{(n, 3)}$-algebra for $\operatorname{dim} \mathcal{A}=n>1$.

\section{THE DISPERSIONLESS $\mathcal{A}-K P$ HIERARCHY}

Because of the similarities in the theories of dispersionless and dispersive KP equations (see Refs. 20 and 23), we list here the analogous results for the $\mathcal{A}$-dKP hierarchy without proofs. We will use the following notation in this part. For an $\mathcal{A}$-valued Laurent series of the form $A=\sum_{i} A_{i} p^{i}$, we denote by $A_{+}$the polynomial part of the Laurent series $A$ and $A_{-}=A-A_{+}$, res $(A)=a_{-1}$. Let

$$
L=\mathbf{1}_{\mathbf{n}} p+U_{1} p^{-1}+U_{2} p^{-2}+\cdots
$$

be an $\mathcal{A}$-valued Laurent series.

Definition 4.1. The $\mathcal{A}-d K P$ hierarchy is the set of equations of motion

$$
\frac{\partial L}{\partial t_{r}}=\left\{L_{+}^{r}, L\right\}
$$

where $\{$,$\} is defined by \{A, B\}=\frac{\partial A}{\partial p} \circ \frac{\partial B}{\partial x}-\frac{\partial A}{\partial x} \circ \frac{\partial B}{\partial p}$. 
Let us assume that $L^{m}, m \in \mathbb{N}$, is of the form

$$
\mathcal{L}:=L^{m}=\mathbf{1}_{\mathbf{n}} p^{m}+V_{m-1} p^{m-1}+\cdots .
$$

Taking a dispersionless limit of Hamiltonian structures for the $\mathcal{A}$-KP hierarchy, we get the first and the second Poisson brackets of the $\mathcal{A}$-dKP hierarchy associated with $\mathcal{L}$ in (4.3) as follows:

$$
\{\tilde{f}, \tilde{g}\}^{m(\infty)}=\operatorname{tr} \int \operatorname{res}\left(\left\{\mathcal{L}_{-},\left(\frac{\delta f}{\delta \mathcal{L}}\right)_{+}\right\}_{-}-\left\{\mathcal{L}_{+},\left(\frac{\delta f}{\delta \mathcal{L}}\right)_{-}\right\}_{+}\right) \circ \frac{\delta g}{\delta \mathcal{L}} d x
$$

and

$$
\{\tilde{f}, \tilde{g}\}^{m(0)}=\operatorname{tr} \int \operatorname{res}\left(\left(\mathcal{L} \circ \frac{\delta f}{\delta \mathcal{L}}\right)_{+} \circ \mathcal{L}-\mathcal{L} \circ\left(\frac{\delta f}{\delta \mathcal{L}} \circ \mathcal{L}\right)_{+}\right) \circ \frac{\delta g}{\delta \mathcal{L}} d x,
$$

where $\tilde{f}, \tilde{g} \in \tilde{\mathcal{D}}$ are two functionals. The variational derivative $\frac{\delta f}{\delta \mathcal{L}}$ is given by

$$
\frac{\delta f}{\delta \mathcal{L}}=\sum_{i=-\infty}^{m-1} \frac{\delta f}{\delta V_{i}} p^{-i-1},
$$

where $\frac{\delta f}{\delta V_{i}}$ is defined in (3.4). When we restrict these to the submanifold $V_{m-1}=0$, the first Hamiltonian structure automatically reduces to this submanifold, but the second one is reducible if and only if

$$
\operatorname{res}\left\{\mathcal{L}, \frac{\delta f}{\delta \mathcal{L}}\right\}=0 \text {. }
$$

Similarly, in terms of the basis $\left\{v_{[i] q}\right\}$, the second Poisson bracket $\{,\}^{m(0)}$ for $L^{m}$ in (4.3) and the reduced bracket $\{,\}_{D}^{m(0)}$ for $L^{m}$ with the constraint $V_{m-1}=0$ will provide two kinds of local $w$-type algebras.

\section{CONCLUSIONS}

In summary, we have introduced the Frobenius algebra-valued KP hierarchy and studied the existence of $\tau$-functions and Hamiltonian structures. Regarding scalar fields as components of a more basic $\mathcal{A}$-valued field is a more elegant approach: it is not basis dependent and it automatically stresses the algebraic properties more clearly. Other properties can then be traced back, for example, to the freedom in the definition of the Frobenius form. Via the properties of the second Hamiltonian structures, we have obtained some local matrix generalizations of $W$-algebras. An interesting byproduct is that the coupled KP hierarchy in Ref. 4 has at least $n$-"basic" different local bi-Hamiltonian structures. The methods in the paper may clearly be applied to other theories of a similar type which have an underlying Lax equation, for example, Toda-hierarchies and reductions of these theories. ${ }^{26,27}$

In a separate paper, $\mathcal{A}$-valued Frobenius manifolds, topological quantum field theories, and bi-Hamiltonian structures are constructed. ${ }^{21}$ These constructions are different in character to those in this paper: they are developed without any use of Lax equations, relying on a "lifting" construction from scalar to algebra-valued fields. There will, clearly, be an overlap, with the theory of $\mathcal{A}$-valued KdV and dKdV equations being the most obvious example.

\section{ACKNOWLEDGMENTS}

D. Zuo is partially supported by No. NCET-13-0550 and NSFC (Nos. 11271345 and 11371338) and the Fundamental Research Funds for the Central Universities.

\footnotetext{
${ }^{1}$ M. Adler, "On a trace functional for formal pseudodifferential operators and the symplectic structure of the Korteweg-de Vries type equations," Invent. Math. 50, 219-248 (1979).

${ }^{2}$ I. Bakas, "Higher spin fields and the Gelfand-Dickey algebra," Commun. Math. Phys. 123, 627-639 (1989).

${ }^{3}$ A. Bilal, "Non-local matrix generalizations of W-algebras," Commun. Math. Phys. 170, 117-150 (1995).
} 
${ }^{4}$ P. Casati and G. Ortenzi, "New integrable hierarchies from vertex operator representations of polynomial Lie algebras," J. Geom. Phys. 56, 418-449 (2006).

${ }^{5}$ E. Date, M. Jimbo, M. Kashiwara, and T. Miwa, "Transformation groups for soliton equations," in Proceedings of R.I.M.S. Symposium on Nonlinear Integrable Systems-Classical Theory and Quantum Theory (World Scientific, 1983), pp. 39-119.

${ }^{6}$ L. A. Dickey, "On Hamiltonian and Lagrangian formalisms for the KP hierarchy of integrable equations," Ann. N. Y. Acad. Sci. 491, 131-148 (1987).

${ }^{7}$ L. A. Dickey, "Lectures on classical W-algebras," Acta Appl. Math. 47, 243-321 (1997).

${ }^{8}$ L. A. Dickey, Soliton Equations and Hamiltonian Systems, Advanced Series in Mathematical Physics Vol. 26, 2nd ed. (World Scientific, 2003).

${ }^{9}$ B. Dubrovin, Geometry of 2D Topological Field Theories, Lecture Notes in Mathematics Vol. 1620 (Springer, 1996), pp. 120-348.

${ }^{10}$ P. Di Franscessco, C. Itzykson, and J. B. Zuber, "Classical W-algebras," Commun. Math. Phys. 140, 543-567 (1991).

${ }^{11}$ A. P. Fordy, A. G. Reyman, and M. A. Semenov-Tian-Shansky, "Classical $r$-matrices and compatible Poisson brackets for coupled KdV systems," Lett. Math. Phys. 17, 25-29 (1989).

12 I. M. Gelfand and L. A. Dickey, "Family of Hamiltonian structures connected with integrable non-linear equations," preprint, IPM, Moscow (1978) (in Russian) [English version in Collected Papers of I. M. Gelfand (Springer-Verlag, 1987), Vol. 1, pp. 625-646].

${ }^{13}$ R. Hirota, X. B. Hu, and X. Y. Tang, "A vector potential KdV equation and vector Ito equation: Soliton solutions, bilinear Bäcklund transformations and Lax pairs.,” J. Math. Anal. Appl. 288(1), 326-348 (2003).

${ }^{14}$ B. A. Kupershmidt, KP or mKP: Noncommutative Mathematics of Lagrangian, Hamiltonian, and Integrable Systems, Mathematical Surveys and Monographs (American Mathematical Society, 2000), Vol. 78.

${ }^{15} \mathrm{~J}$. van de Leur, "Bäcklund transformations for new integrable hierarchies related to the polynomial Lie algebra $g l_{\infty}^{(n)}$," J. Geom. Phys. 57, 435-447 (2007).

${ }^{16}$ W. X. Ma and B. Fuchssteinery, “The bi-Hamiltonian structure of the perturbation equations of KdV hierarchy," Phys. Lett. A 213, 49-55 (1996).

${ }^{17}$ P. J. Olver and V. V. Sokolov, "Integrable evolution equations on associative algebras," Commun. Math. Phys. 2, 245-268 (1998).

18 A. O. Radul, "Two series of Hamiltonian structures for the hierarchy of Kadomtsev-Petviashvili equations," in Applied Methods of Nonlinear Analysis and Control, edited by Mironov, Moroz, and Tshernyatin (Moscow State University, Moscow, 1987), pp. 149-157.

${ }^{19}$ M. Sato, "Soliton equations as dynamical systems on infinite dimensional Grassmann manifolds," RIMS Kokyuroku 439 , 30-46 (1981).

${ }^{20}$ I. A. B. Strachan, “The Moyal bracket and the dispersionless limit of the KP hierarchy,” J. Phys. A: Math. Gen. 28, 1967-1975 (1995).

${ }^{21}$ I. A. B. Strachan and D. Zuo, "Frobenius manifolds and Frobenius algebra-valued integrable systems," preprint arXiv: 1403.0021.

22 Y. Watanabe, "Hamiltonian structure of Sato's hierarchy of KP equations and a coadjoint orbit of a certain formal Lie group," Lett. Math. Phys. 7(2), 99-106 (1983).

${ }^{23}$ D. Zuo, "On the Kuperschmidt-Wilson theorem for the Moyal-Kadomtsev-Petviasfvili hierarchy," Inverse Probl. 22, 1959-1966 (2006).

${ }^{24}$ D. Zuo, "Local matrix generalizations of W-algebras" (unpublished); e-print arXiv:1401.2216v1.

${ }^{25}$ D. Zuo, "The Frobenius-Virasoro algebra and Euler equations," J. Geom. Phys. 86, 203-210 (2014).

${ }^{26}$ H. Zhang and D. Zuo, "Hamiltonian structures of the constrained $\mathcal{F}$-valued KP hierarchy," Rep. Math. Phys. 76, 116-129 (2015).

${ }^{27} \mathrm{~K}-\mathrm{L}$. Tian and D. Zuo, "Free field realizations of some local matrix W-algebras" (unpublished).

${ }^{28}$ A skew mapping $\mathcal{H}: \Omega^{1} \rightarrow \mathcal{V}$ is said to be Hamiltonian if (1) $\mathcal{H} \Omega^{1} \subset \mathcal{V}$ is a Lie subalgebra and (2) the 2-form $\omega$ defined by $\omega(\mathcal{H}(X), \mathcal{H}(Y))=\langle\mathcal{H}(X), Y\rangle$ is closed. 